\title{
THE LATIN SQUARE, OR CYCLIC, FUNCTIONS*
}

BY

E. T. BELL

1. Introduction. Special cases of the Latin square functions defined in this paper have recently come into some prominence in connection with generalizations by Humbert and others (references in \$5) of the partial differential equations of mathematical physics. In solving the equations, the functions of $r-1$ independent variables defined by Appell ${ }^{3} \dagger$ in 1877 appear, and these in turn are intimately connected with Olivier's ${ }^{1} \dagger$ functions $f_{0}(x), \cdots, f_{r-1}(x)$, whose generating identity is

$$
\exp \alpha x=f_{0}(x)+\alpha f_{1}(x)+\cdots+\alpha^{r-1} f_{r-1}(x),
$$

where $\alpha$ is an imaginary $r$ th root of unity,

$$
f_{j}(x)=\sum x^{n_{i}} / n_{j} !
$$

the summation referring to all integers $n_{j} \geqq 0$ such that $n_{j} \equiv j \bmod r$. We shall call $r$ the base of $f_{j}(n)$. Appell's functions $A_{t}$ can be defined by expanding the left member of the following identity as a power series in $\alpha$, and reducing the result modulo $\alpha^{r}-1$,

$$
\exp \left(\sum_{s=1}^{r-1} \alpha^{s} x_{s}\right)=\sum_{t=0}^{r-1} \alpha^{t} A_{t}\left(x_{1}, \cdots, x_{r-1}\right) .
$$

The $r$ functions $A_{t}\left(x_{1}, \cdots, x_{r-1}\right) \equiv A_{t}$ are connected by the identical algebraic relation

$$
N\left(A_{0}, \cdots, A_{r-1}\right)=1,
$$

where $N\left(y_{0}, \cdots, y_{r-1}\right)$ is the norm of the algebraic number

$$
y_{0}+\alpha y_{1}+\cdots+\alpha^{r-1} y_{r-1} \text {. }
$$

As the partial differential equations mentioned have no immediate physical significance, there is no apparent reason for stopping short of the general case. In a previous paper ${ }^{7}$ the functions defined by reducing the left of (3) modulo $P(\alpha)$, where $P(\alpha)$ is any polynomial in $\alpha$, were introduced and some of their properties discussed. The norm property (1.4) does not hold for these functions, except in the very degenerate case when they become Appell's

* Presented to the Society, March 18, 1933; received by the editors December 27, 1932.

$\dagger$ Numbers refer to bibliography in $\S 5$. 
It will be interesting to see what replaces the norm property, and how it degenerates in the special case.

We shall see that the generalized norm property is intimately connected with Latin squares. A Latin square of degree $n$ is a square array of $n$ distinct elements such that no element occurs twice in the same column. The number of Latin squares of degree $n$, no two of which can be derived from one another by a permutation of rows or of columns, will be denoted by $\lambda(n)$. This number has not been determined for general $n$, and even for small $n$ the labor of a direct determination is prohibitive (see MacMahon ${ }^{6}$ ). As observed by Cayley, ${ }^{5}$ not every Latin square of given degree can be generated by a group of substitutions on the elements of a given row. Thus there exist (even for $n$ small) Latin squares with which no group is associated.

The norm relation is replaced for the generalized functions of $r$ independent variables by $\lambda(r)$ algebraic relations, each of which is derived from a Latin square of degree $r$. When the functions degenerate to Appell's (based on $r$ th roots of unity), the $\lambda(r)$ relations coalesce in the norm relation, and the single Latin square corresponding to this relation is generated from its first row by the cyclic group of degree $r$.

Appell's functions are a simple generalization to functions of $r$ independent variables of the circular and hyperbolic functions. The Latin square functions pass at once to the most general situation possible of this kind, namely to the functions of $r$ independent variables constructed from polynomials in the members of sets of $r$ linearly independent solutions of equations of the type

$$
\frac{d^{r} y}{d x^{r}}+c_{1} \frac{d^{r-1} y}{d x^{r-1}}+\cdots+c_{r} y=0
$$

where $c_{1}, \cdots, c_{r}$ are arbitrary constants, instead of from the degenerate case $c_{r}=-1, c_{j}=0, j \neq r$. The coefficients in the power series for Olivier's functions, on which Appell's are based, are periodic. In the generalized functions periodicity, $\phi(n+r)=\phi(n)$ for all integers $n$, is replaced by

$$
\phi(n+r)+c_{1} \phi(n+r-1)+\cdots+c_{r} \phi(n)=0,
$$

which becomes periodicity in the degenerate case.

All the functions defined are obviously continuous and convergent absolutely for all finite values of the variables.

2. Generalized Olivier functions. Consider first the generalization of Olivier's functions. Let

$$
P(\alpha) \equiv \alpha^{r}+c_{1} \alpha^{r-1}+\cdots+c_{r}
$$

be irreducible in the rational domain. Reduction modulo $P(\alpha)$ of the expan- 
sion of $\exp \alpha^{s} x$, where $s$ is an integer, defines the functions $f_{j}\left(\begin{array}{l}x \\ s\end{array}\right)$ uniquely,

$$
\exp \alpha^{s} x=\sum_{j=0}^{r-1} \alpha^{j} f_{j}\left(\begin{array}{l}
x \\
s
\end{array}\right)
$$

since $P(\alpha)$ is irreducible. We write

$$
f_{i}\left(\begin{array}{l}
x \\
1
\end{array}\right) \equiv f_{j}(x) \quad(j=0, \cdots, r-1) .
$$

The notation in (2.1) is fixed throughout the paper.

The $j$ th fundamental sequence $\phi_{j}(n), n=0, \pm 1, \pm 2, \cdots$, defined by the difference equation

$$
\phi(n+r)+c_{1} \phi(n+r-1)+\cdots+c_{r} \phi(n)=0,
$$

whose characteristic equation is $P(\alpha)=0$, is determined by

$$
\phi_{j}(k)=\delta_{j}{ }^{k} \text { (Kronecker delta), } j, k=0, \cdots, r-1 .
$$

The $\phi_{j}(n)$ are a set of $r$ linearly independent solutions of (2.4), and the general solution $\phi(n)$ is

$$
\phi(n)=\sum_{j=0}^{r-1} \phi(j) \phi_{j}(n) .
$$

The notations in (2.4), (2.5) are fixed henceforth.

For all integers $n$ we have

$$
\alpha^{n}=\sum_{j=0}^{r-1} \alpha^{j} \phi_{j}(n)
$$

Hence, by (2.2),

$$
f_{j}\left(\begin{array}{l}
x \\
s
\end{array}\right)=\sum_{n=0}^{\infty} \phi_{j}(s n) \frac{x^{n}}{n !} \quad(j=0, \cdots, r-1) .
$$

To find the differential equation satisfied by the functions (2.8), let $P_{s}(\alpha)$ be the polynomial with leading coefficient unity whose roots are the sth powers of the roots of $P_{1}(\alpha) \equiv P(\alpha)$,

$$
P_{s}(\alpha) \equiv \beta^{r}+c_{1}(s) \beta^{r-1}+\cdots+c_{r}(s) \quad\left(\beta \equiv \alpha^{s}\right) .
$$

Then, by (2.2), (2.9), $r$ linearly independent solutions of

$$
\frac{d^{r} y}{d x^{r}}+c_{1}(s) \frac{d^{r-1} y}{d x^{r-1}}+\cdots+c_{r}(s) y=0
$$

are the functions (2.8), and the general solution of (2.10) is 


$$
y=\sum_{j=0}^{r-1} k_{i} f_{j}\left(\begin{array}{l}
x \\
s
\end{array}\right),
$$

where the $k_{j}$ are arbitrary constants.

The exponential forms of the functions (2.8), corresponding to those of the circular, hyperbolic, and Olivier functions, are obtained at once from (2.2). If $\alpha_{0}, \cdots, \alpha_{r-1}$ are the roots of $P(\alpha)=0$, and $\alpha^{i j}$ denotes the cofactor of $\alpha_{i}^{j}$ in the determinant

we have

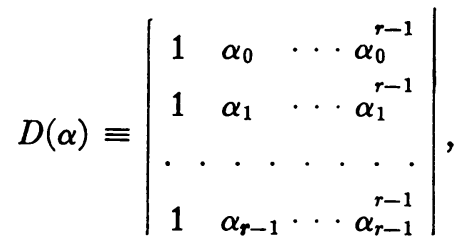

$$
D(\alpha) f_{j}\left(\begin{array}{c}
x \\
s
\end{array}\right)=\sum_{t=0}^{r-1} \alpha^{t j} \exp \alpha_{t} x^{s}
$$

since $D(\alpha) \neq 0, P(\alpha)$ being irreducible.

Corresponding to the period recurrence of the derivatives of the circular, hyperbolic, and Olivier functions, we have here

$$
\frac{d^{t}}{d x^{t}} f_{k}\left(\begin{array}{l}
x \\
s
\end{array}\right)=\sum_{j=0}^{r-1} \phi_{k}(s t+j) f_{j}\left(\begin{array}{l}
x \\
s
\end{array}\right) \quad(k=0, \cdots, r-1),
$$

on differentiating (2.2) $t$ times and applying (2.7).

Applying (2.7) to the product of $\exp \alpha^{s} x$ and $\exp \alpha^{s} y$, we get the addition theorems

$$
f_{j}\left(\begin{array}{c}
x+y \\
s
\end{array}\right)=\sum_{p=0}^{r-1} \sum_{k=0}^{r-1} \phi_{i}(p+k) f_{p}\left(\begin{array}{l}
x \\
s
\end{array}\right) f_{k}\left(\begin{array}{l}
y \\
s
\end{array}\right) .
$$

There is no algebraic addition theorem with respect to $s$.

Let $\alpha$ be any root of $P(\alpha)=0$. Then

$$
\exp \left[x \alpha^{8} P(\alpha)\right]=1,
$$

and hence, by (22), the identical algebraic relations between the functions are obtained by reducing the expression on the left of the following, modulo $P(\alpha)$, to that on the right $\left(c_{0}=1\right)$,

$$
\prod_{k=0}^{r-1}\left[\sum_{j_{k}=0}^{r-1} \alpha^{j_{k}} f_{j_{k}}\left(\begin{array}{c}
c_{k} x \\
s+r-k
\end{array}\right)\right]=\sum_{j=0}^{r-1} \alpha^{i} N_{j}\left(\begin{array}{l}
x \\
s
\end{array}\right) ;
$$

the relations are 


$$
N_{0}\left(\begin{array}{l}
x \\
s
\end{array}\right)=1, \quad N_{p}\left(\begin{array}{l}
x \\
s
\end{array}\right)=0 \quad(p=1, \cdots, r-1)
$$

For Olivier's functions it is easily seen that (2.16) are equivalent to the single norm relation (the last $r-1$ relations are absent).

If $P(\alpha)$ is such that, for some integer $s>0, c_{1}(s)=0$ in (2.9), the functions $f_{j}\left(\begin{array}{l}x \\ s\end{array}\right), j=0, \cdots, r-1$, are more simply connected. Let the roots of $P_{s}(\alpha)=0$ be $\beta_{0}, \cdots, \beta_{r-1}$. If $\beta$ is any one of the roots, we may define functions $g_{j}\left(\begin{array}{l}x \\ l\end{array}\right)$ by the process for (2.2) with $P(\alpha)$ replaced by the right of (2.9),

$$
\exp \beta^{t} x=\sum_{j=0}^{r-1} \beta^{i} g_{j}\left(\begin{array}{l}
x \\
t
\end{array}\right) .
$$

Apply (2.7) to $\beta^{i} \equiv \alpha^{j s}$. Then

$$
\begin{gathered}
f_{k}\left(\begin{array}{l}
x \\
s t
\end{array}\right)=\sum_{j=0}^{r-1} \phi_{k}(j s) g_{j}\left(\begin{array}{l}
x \\
t
\end{array}\right) \quad(k=0, \cdots, r-1) ; \\
g_{j}\left(\begin{array}{l}
x \\
1
\end{array}\right) \equiv g_{j}(x) .
\end{gathered}
$$

If now $c_{1}(s)=0, \beta_{0}+\cdots+\beta_{r-1}=0$, and $\exp \left(\beta_{0}+\cdots+\beta_{r-1}\right) x=1$. Hence

$$
M_{0}\left(g_{0}(x), \cdots, g_{+-1}(x)\right)=1 \text {, }
$$

where $M_{0}\left(y_{0}, \cdots, y_{r-1}\right)$ is the norm of $y_{0}+y_{1} \beta+\cdots+y_{r-1} \beta^{r-1}$. If further $s=1,(2.16)$ hence becomes

$$
N_{0}\left(f_{0}(x), \cdots, f_{r-1}(x)\right)=1,
$$

where $N_{0}\left(y_{0}, \cdots, y_{r-1}\right)$ is the norm of $y_{0}+y_{1} \alpha+\cdots+y_{r-1} \alpha^{r-1}$. A linear transformation on the $f_{i}(x)$ will always reduce $(2.16)$ when $s=1$ to $(2.20)$.

Since there are precisely $r$ functions $f_{j}\left(\begin{array}{l}x \\ s\end{array}\right)$ of the single variable $x$, they must be connected by $r-1$ relations. These are contained in the $r$ relations (2.12), which are not independent, or in the equivalent dependent set obtained from (2.2) by putting $\alpha_{0}, \cdots, \alpha_{r-1}$ successively for $\alpha$. The dependence for the last set of $r$ is evident from $-c_{1}(s)=\alpha_{0}{ }^{8}+\cdots+\alpha_{r-1}^{s} ; c_{1}(s)$ is a rational function of $c_{1}, \cdots, c_{r-1}$. The $r-1$ independent relations are transcendental.

3. Functions with periodic coefficients. A special case of the functions (2.3) is of particular interest as it can be completely specified with remarkable simplicity. In a previous note ${ }^{10}$ it was shown that the only difference equations (2.4) whose solutions have the proper additive period $m$ (integer $>0$ ) are those in which $r=\tau(m)$, the totient (Euler's function) of $m$, and $P(\alpha)=0$ is 
the equation whose roots are the $\tau(m)$ primitive $m$ th roots of unity. In this section $m$ is a constant integer $>0, r=\tau(m)$, and

$$
\alpha^{r}+c_{1} \alpha^{r-1}+\cdots+c_{r}=0
$$

is the equation for the primitive $m$ th roots of unity. All of $\$ 2$ necessarily holds in this case, with special features not valid in \$2. The notation is as before; in particular the general solution of

$$
\phi(n+r)+c_{1} \phi(n+r-1)+\cdots+c_{r} \phi(n)=0
$$

is $\phi(n)$. The sequence $\phi(n)(n=0, \pm 1, \cdots)$ is determined by (3.2) when $\phi(0), \cdots, \phi(r-1)$ are given constants.

From what has just been recalled it follows that the only functions

$$
f(x) \equiv \sum_{n=0}^{\infty} \psi(n) x^{n} / n !
$$

in which $\psi(n)$ has the proper additive period $m$ and is determined by a linear difference equation with constant coefficients, are those in which $\psi=\phi$,

and hence

$$
f(x)=\sum_{n=0}^{\infty} \phi(n) x^{n} / n !
$$

$$
f(x)=\sum_{t=0}^{m-1} \phi(t)\left[\sum_{n=0}^{\infty} \frac{x^{n+t}}{(n m+t) !}\right] .
$$

The functions in square brackets, say

$$
h_{t}(x) \equiv \sum_{n=0}^{\infty} \frac{x^{n m+t}}{(n m+t) !},
$$

are the $m$ Olivier functions to the base $m$; see (1.2). Hence, by (2.6), the general function (3.3) with periodic coefficients of the kind described is

$$
f(x)=\sum_{j=0}^{r-1} \phi(j)\left[\sum_{t=0}^{m-1} \phi_{j}(t) h_{t}(x)\right] .
$$

Consider the functions in the square brackets in (3.6),

$$
H_{j}(x) \equiv \sum_{t=0}^{m-1} \phi_{j}(t) h_{t}(x) \quad(j=0, \cdots, r-1) .
$$

The generating identity is

$$
\exp \alpha x=\sum_{j=0}^{r-1} \alpha^{i} H_{j}(x)
$$


where $\alpha$ is any root of (3.1). Hence, taking the $n$th derivative, we get

$$
\frac{d^{n}}{d x^{n}} H_{j}(x)=\sum_{i=0}^{r-1} \phi_{j}(n+i) H_{i}(x) ;
$$

and therefore, by the periodicity of $\phi_{j}$,

$$
\begin{aligned}
\frac{d^{k m+n}}{d x^{k m+n}} H_{j}(x) & =\frac{d^{n}}{d x^{n}} H_{j}(x) \\
(n & =0, \cdots, m-1 ; j=0, \cdots, r-1)
\end{aligned}
$$

for all integers $k \geqq 0$. Thus the derivatives of the $H_{j}(x)$ recur with the period $m$. Since $\phi(0), \cdots, \phi(r-1)$ in (3.6) are arbitrary constants, (3.9) implies

$$
\frac{d^{k m+n}}{d x^{k m+n}} f(x)=\frac{d^{n}}{d x^{n}} f(x),
$$

and $f(x)$ is the most general function with recurring derivatives of period $m$.

Consider next the functions (3.3) in which $\psi(n)$ has the proper multiplicative period $m+1$ ( $m$ integer $>0)$, and in which $\psi(n)$ is determined by a linear difference equation with constant coefficients. It follows from the theorem recalled for additive periodicity that the only such $\psi(n)$ with multiplicative period $m+1>1$ are the $\phi(n)$ defined by (3.1) as before. Hence the properties of these functions follow from those just discussed.

4. Latin square functions. In this section the notation is as in $\$ 2$. We shall need particularly (2.1)-(2.4). As a basis for the numbers of the field $K(\alpha)$ we shall take $1, \alpha, \cdots, \alpha^{r-1}$, and we shall denote the element of $K(\alpha)$ whose coordinates are $x_{0}, \cdots, x_{r-1}$ by $(x)$,

$$
(x) \equiv\left(x_{0}, \cdots, x_{r-1}\right) \equiv x_{0}+\alpha x_{1}+\cdots+\alpha^{r-1} x_{r-1} .
$$

The sum of $(x),(y)$ may be written either as $(x)+(y)$ or $(x+y)$,

$$
(x+y)=\left(x_{0}+y_{0}, \cdots, x_{r-1}+y_{r-1}\right) ;
$$

their product, $(x)(y)$ or $(x y)$, is

$$
\begin{aligned}
(x y) & =\left((x y)_{0}, \cdots,(x y)_{r-1}\right), \\
(x y)_{j} & \equiv \sum_{i=0}^{r-1} \sum_{p=0}^{r-1} \phi_{j}(i+p) x_{i} y_{p} .
\end{aligned}
$$

More generally, the product of any finite number of elements $(x),(y), \cdots$, $(z)$ of $K(\alpha)$, in a similar notation, is given by

$$
\begin{aligned}
&(x y \cdots z)_{j} \equiv \sum \phi_{j}(i+p+\cdots+t) x_{i} y_{p} \cdots z_{t}, \\
& 0 \leqq i, p, \cdots, t \leqq r-1 .
\end{aligned}
$$


The element $(x)^{\prime}$ of $K(\alpha)$ defined by

$$
\begin{aligned}
& (x)^{\prime} \equiv(x)-x_{0}+x_{r} \alpha^{r} \equiv\left(x_{0}^{\prime}, \cdots, x_{r-1}^{\prime}\right), \\
& x_{0}^{\prime}=-c_{r} x_{r}, x_{k}^{\prime}=x_{k}-c_{r-k} x_{r} \quad(k=1, \cdots, r-1), \\
& (x)^{\prime}=\alpha x_{1}+\alpha^{2} x_{2}+\cdots+\alpha^{r} x_{r}
\end{aligned}
$$

will be called the curtate of $(x)$. Accents as in $(x)^{\prime},(y)^{\prime}, \cdots$ shall denote the curtates of the corresponding $(x),(y), \ldots$.

The Latin square functions of degree $r$ in the independent variables $x_{1}$, $\cdots, x_{r}$ are denoted by $L_{j}\left(x_{1}, \cdots, x_{r}\right)$, and are defined by the identity (4.6), in which the right is the reduction modulo $P(\alpha)$ of the expansion of the left as a power series in $\alpha$,

$$
\exp (x)^{\prime} \equiv \sum_{j=0}^{r-1} \alpha^{j} L_{j}\left(x_{1}, \cdots, x_{r}\right) .
$$

To find the algebraic relations between the $L_{j}$ mentioned in $\$ 1$, we proceed as described presently from the Latin square (4.7) to its "bordered mate" (4.8). We assume $r \geqq 1$. Let $x_{1}^{(i)}, \cdots, x_{r}^{(i)}$ be the $i$ th row of the Latin square (4.7) of degree $r$ constructed from $x_{1}, \cdots, x_{r}$, so that $x_{1}^{(i)}, \cdots, x_{r}^{(i)}$ is some permutation of $x_{1}, \cdots, x_{r}$ :

$$
\begin{aligned}
& x_{1}^{(1)}, x_{2}^{(1)}, \cdots, x_{r}^{(1)} \\
& x_{1}^{(2)}, x_{2}^{(2)}, \cdots, x_{r}^{(2)} \\
& \cdot \cdot \cdot \cdot \cdot \cdot \cdot \cdot \cdot \\
& x_{1}^{(r)}, x_{2}^{(r)}, \cdots, x_{r}^{(r)} .
\end{aligned}
$$

Write $-s \equiv x_{1}+x_{2}+\cdots+x_{r}$. Multiply the elements in the $j$ th column, $j>1$, of (4.7) by $c_{j-1}$. Apply $\alpha^{r}, \alpha^{r-1}, \ldots, \alpha$ as top border to the result, and $s, c_{1} s, c_{2} s, \cdots, c_{r-1} s$ as a bottom border:

$$
\begin{aligned}
& \frac{\alpha^{r}, \quad \alpha^{r-1}, \cdots, \alpha}{x_{1}^{(1)}, c_{1} x_{2}^{(1)}, \cdots, c_{r-1} x_{r}^{(1)}} \\
& x_{1}^{(2)}, c_{1} x_{2}^{(2)}, \cdots, c_{r-1} x_{r}^{(2)} \\
& \frac{x_{1}^{(r)}, c_{1} x_{2}^{(r)}, \cdots, c_{r-1} x_{r}^{(r)}}{s, \quad c_{1} s, \cdots, c_{r-1} s}
\end{aligned}
$$

Consider the rows of (4.8) as vectors and take the inner product of the vector whose coordinates are the top border by each of the remaining $r+1$. The sum of these $r+1$ inner products vanishes, as it is $\left(x_{1}+\cdots+x_{r}\right) P(\alpha)$, from the 
construction of (4.8). These products are the curtates

$$
\begin{aligned}
& \left(c_{r-1} x_{r}^{(i)}, c_{r-2} x_{r-1}^{(i)}, \cdots, c_{1} x_{2}^{(i)}, x_{1}^{(i)}\right)^{\prime} \quad(i=1, \cdots, r), \\
& \left(c_{r-1} s, c_{r-2} s, \cdots, c_{1} s, s\right)^{\prime} .
\end{aligned}
$$

To simplify the writing, let the $r+1$ curtates in (4.9) be equal respectively to

$$
\begin{array}{ll}
\left(y_{1}^{(i)}, y_{2}^{(i)}, \cdots, y_{r}^{(i)}\right)^{\prime} & (i=1, \cdots, r), \\
\left(y_{1}^{(r+1)}, y_{2}^{(r+1)}, \cdots, y_{r}^{(r+1)}\right)^{\prime} . &
\end{array}
$$

Then

$$
\exp \left[\sum_{p=1}^{r+1}\left(y_{1}^{(p)}, \cdots, y_{r}^{(p)}\right)^{\prime}\right]=1
$$

and hence, by (4.6),

$$
\prod_{p=1}^{r+1}\left[\sum_{j_{p=0}}^{r-1} \alpha^{j_{p}} L_{i_{p}}\left(y_{1}^{(p)}, \cdots, y_{r}^{(p)}\right)\right]=1 .
$$

When distributed and reduced modulo $P(\alpha)$, the left of (4.11) is of the form $N_{0}+\alpha N_{1}+\cdots+\alpha^{r-1} N_{r-1}$, where $N_{j}$ is a homogeneous polynomial of degree $r+1$ in functions $L_{0}, L_{1}, \cdots, L_{r-1}$ whose variables are given in (4.10). For the moment the structure of the $N_{j}$ need not be considered. Starting then with the particular Latin square (4.7), we reach the identical relations

$$
N_{0}=1, N_{j}=0 \quad(j=1, \cdots, r-1) .
$$

We indicate the structure of the $N$ 's presently.

From (4.5), (4.6) we find explicit forms for the $L_{j}$. The expression for the $L_{j}$ corresponding to $(2.12)$ is obvious and can be omitted. Let $\theta_{0}, \cdots, \theta_{r-1}$ be the $r$ conjugates of $(x)^{\prime}$, including $(x)^{\prime}$. Form the equation

$$
\theta^{r}+b_{1} \theta^{r-1}+\cdots+b_{r}=0 \quad\left(\theta=\theta_{0}, \cdots, \theta_{r-1}\right)
$$

whose roots are these conjugates. Then $b_{j} \equiv b_{j}\left(x_{1}, \cdots, x_{r}\right)$ is a homogeneous polynomial of degree $j$ in $x_{1}, \cdots, x_{r}$, whose coefficients are polynomials in $c_{1}, \cdots, c_{r}$ with rational integer coefficients. Similarly to the discussion for (2.3) -(2.5) we consider the difference equation

$$
\xi(n+r)+b_{1} \xi(n+r-1)+\cdots+b_{r} \xi(n)=0,
$$

whose characteristic equation is (4.121). The $r$ fundamental sequences $\xi_{j}(n)$ for (4.13) are determined by

$$
\xi_{j}(k)=\delta_{j}^{k} \quad(j, k=0, \cdots, r-1)
$$


the general solution $\xi(n)$ is

$$
\xi(n)=\sum_{j=0}^{r-1} \xi(j) \xi_{j}(n)
$$

and we have

$$
\theta^{n}=\sum_{j=0}^{r-1} \theta^{i} \xi_{j}(n)
$$

The powers of $\theta$ on the right of (4.16) must be reduced modulo $P(\alpha)$ independently. Let

$$
\theta^{i}=p_{j 0}+\alpha p_{j 1}+\cdots+\alpha^{r-1} p_{j, r-1} \quad(j=1, \cdots, r-1) .
$$

Then $p_{j i} \equiv p_{j i}\left(x_{1}, \cdots, x_{r}\right)$ is a polynomial in $x_{1}, \cdots, x_{r}$ whose coefficients are polynomials in $c_{1}, \cdots, c_{r}$ with rational integer coefficients. From (4.16) we now have

$$
\theta^{n}=\sum_{j=0}^{r-1} \alpha^{j}\left[\sum_{i=0}^{r-1} p_{i j} \xi_{i}(n)\right]
$$

hence, by (4.6),

$$
L_{j}\left(x_{1}, \cdots, x_{r}\right)=\sum_{i=0}^{r-1} p_{i j} \sum_{n=0}^{\infty} \frac{\xi_{i}(n)}{n !} \quad(j=0, \cdots, r-1) .
$$

The $p_{i j}$ are defined by (4.17), and the $\xi_{j}(n)$ are the fundamental solutions of (4.13).

Since the variables $x_{1}, \cdots, x_{r}$ are independent, the differential relations of $\$ 2$ go over, by (4.6), to corresponding relations for the $L_{j}$. Thus from (2.10), (4.6) we have

$$
\begin{array}{r}
{\left[\frac{\partial^{r}}{\partial x_{s}^{r}}+c_{1}(s) \frac{\partial^{r-1}}{\partial x_{s}^{r-1}}+\cdots+c_{r}(s)\right] L_{j}\left(x_{1}, \cdots, x_{r}\right)=0} \\
(j=0, \cdots, r-1 ; s=1, \cdots, r)
\end{array}
$$

and corresponding to (2.13),

$$
\frac{\partial^{t}}{\partial x_{s}^{t}} L_{k}\left(x_{1}, \cdots, x_{r}\right)=\sum_{j=0}^{r-1} \phi_{k}(s t+j) L_{j}\left(x_{1}, \cdots, x_{r}\right)
$$

whence

$$
\left(\frac{\partial^{m}}{\partial x_{n}^{m}}-\frac{\partial^{n}}{\partial x_{m}^{n}}\right) L_{k}\left(x_{1}, \cdots, x_{r}\right)=0 \quad(m, n=1, \cdots, r)
$$


The expressions for the $L_{k}$ as polynomials in the functions defined in (2.8) follow at once from (4.4)-(4.6),

$$
L_{k}\left(x_{1}, \cdots, x_{r}\right)=\sum \phi_{k}\left(j_{0}+\cdots+j_{r-1}\right) f_{j_{0}}\left(\begin{array}{c}
x_{0}^{\prime} \\
0
\end{array}\right) \cdots f_{i_{r-1}}\left(\begin{array}{c}
x_{r-1}^{\prime} \\
r-1
\end{array}\right),
$$

the sum extending to all $0 \leqq j_{0}, \cdots, j_{r-1} \leqq r-1$. The $N$ 's in (4.12) have a similar structure in terms of $L$ 's. The addition theorems are of the same type, but simpler,

$$
F_{t}\left(x_{1}+y_{1}, \cdots, x_{r}+y_{r}\right)=\sum \phi_{t}(j+k) F_{j}\left(x_{1}, \cdots, x_{r}\right) F_{k}\left(y_{1}, \cdots, y_{r}\right),
$$

summed for $0 \leqq j, k \leqq r-1$.

From this point on, the connection with partial differential equations is of the same kind as that for the Appell functions and the equations discussed by Humbert and others in the papers cited in $\$ 5$. The note ${ }^{14}$ sufficiently indicates the start.

5. References. Several of the following papers contain further references to the literature of Appell's functions and their connection with differential equations. The references are given in chronological order. Humbert (loc. cit., p. 153) attributes Olivier's functions to Yvon Villarceau, without stating the reference.

1. L. Olivier, Bemerkungen über eine Art von Funktionen . . ., Crelle's Journal, vol. 2 (1827), pp. 243-251.

2. J. W. L. Glaisher, On functions with recurring derivatives, Proceedings of the London Mathematical Society, vol. 4 (1872), pp. 113-116.

3. P. Appell, Sur certaines fonctions analogues aux fonctions circulaires, Comptes Rendus, Paris, vol. 84 (1877), pp. 1378-1380.

4. J. W. L. Glaisher, Functions analogous to the sine and cosine, Quarterly Journal, vol. 16 (1879), pp. 15-33.

5. A. Cayley, On Latin squares, Messenger of Mathematics, vol.19 (1890), pp. 135-137 (Collected Papers, vol. 13, No. 903).

6. P. A. MacMahon, Combinatorial Analysis, vol. 1, 1915.

7. E. T. Bell, Periodic functions of $n$ variables connected with an algebraic number field of degree n, Quarterly Journal, vol. 50 (1927), pp. 314-328.

8. P.Humbert, Sur une généralisation de l'équation de Laplace, Journal des Mathématiques, (9), vol. 8 (1929), pp. 145-159.

9. D. V. Jonescu, Sur une équation aux dérivées partielles du troisième ordre, Bulletin de la Société Mathématique de France, vol. 58 (1930), pp. 224-229.

10. E. T. Bell, Periodic recurring series, Proceedings of the National Academy of Sciences, vol. 16 (1930), pp. 750-752. 
11. J. Devisme, Comptes Rendus, Paris, vol. 193 (1931), pp. 981-983, 825-828; ibid., vol. 194, pp. 516-519.

12. P. Humbert, On Appell's function $P(\theta, \phi)$, Proceedings of the Edinburg Mathematical Society, (2), vol. 3(1932), pp. 53-55.

13. J. Devisme, Sur la fonction génératrice de la fonction $P(m \theta, n \phi)$ d'Appell, Académie Royale de Belgique, Bulletin, (5), vol. 18 (1932), pp. 505-506.

14. E. T..Bell, $A$ Laplacian equation, American Mathematical Monthly, vol. 39 (1932), pp. 515-517.

California Institute of Technology,

Pasadena, Calir. 